\title{
VECTOR FIELDS WHOSE LINEARISATION IS HURWITZ ALMOST EVERYWHERE
}

\author{
BENITO PIRES AND ROLAND RABANAL
}

(Communicated by Yingfei Yi)

Dedicated to the memory of Professor Carlos Gutierrez

\begin{abstract}
A real matrix is Hurwitz if its eigenvalues have negative real parts. The following generalisation of the Bidimensional Global Asymptotic Stability Problem (BGAS) is provided. Let $X: \mathbb{R}^{2} \rightarrow \mathbb{R}^{2}$ be a $C^{1}$ vector field whose Jacobian matrix $D X(p)$ is Hurwitz for Lebesgue almost all $p \in \mathbb{R}^{2}$. Then the singularity set of $X$ is either an empty set, a one-point set or a non-discrete set. Moreover, if $X$ has a hyperbolic singularity, then $X$ is topologically equivalent to the radial vector field $(x, y) \mapsto(-x,-y)$. This generalises BGAS to the case in which the vector field is not necessarily a local diffeomorphism.
\end{abstract}

\section{INTRODUCTION}

This paper provides sufficient conditions for a planar $C^{1}$ vector field to be globally asymptotically stable, that is, to be topologically equivalent to the radial vector field $(x, y) \mapsto(-x,-y)$. The strongest result in this respect is due to Feßler [1], Glutsyuk [3] and Gutierrez [4] who solved positively the Markus-Yamabe Problem [7. (also known as the Bidimensional Global Asymptotic Stability Problem):

Theorem 1.1 (BGAS). Let $X: \mathbb{R}^{2} \rightarrow \mathbb{R}^{2}$ be a $C^{1}$ vector field such that $X(0)=0$. If its Jacobian matrix $D X(p)$ is Hurwitz for every $p \in \mathbb{R}^{2}$, then $X$ is globally asymptotically stable.

The condition that $D X(p)$ is Hurwitz is equivalent to Trace $(D X(p))<0$ and $\operatorname{Det}(D X(p))>0$. Thus Theorem 1.1 can only be applied to vector fields that are local diffeomorphisms. However, it is not difficult to find globally asymptotically stable vector fields whose Jacobian matrix is Hurwitz everywhere except along curves or points where its determinant or its trace vanishes. For example, the Jacobian matrix of the planar vector field $X: \mathbb{R}^{2} \rightarrow \mathbb{R}^{2}$ defined by

$$
X(x, y)=\left(-(x+1)^{3}+1,-(x+1)^{2}(y+1)+1\right)
$$

is Hurwitz Lebesgue almost everywhere but not everywhere. Moreover, $X(0)=0$. We cannot apply Theorem 1.1, but we can apply Theorem A to conclude that $X$ is globally asymptotically stable. Notice that this vector field (as a map) is neither a local diffeomorphism nor an injective map. Therefore, the aim of this paper is to

Received by the editors November 29, 2011 and, in revised form, September 21, 2012.

2010 Mathematics Subject Classification. Primary 34D23, 37B25; Secondary 37C10.

The first author was partially supported by FAPESP-Brazil (2009/02380-0 and 2008/02841-4).

The second author was partially supported by PUCP-Peru (DAI 2010-0058) and ICTPItaly (220 (Maths) RR/ab). 
provide a generalisation of Theorem 1.1 which includes vector fields which are not necessarily local diffeomorphisms.

This paper also contains a result (Theorem B) about the cardinality of the singularity set of a $C^{1}$ vector field whose Jacobian matrix is Hurwitz at almost all points of $\mathbb{R}^{2}$. It states that under such a hypothesis the singularity set of the vector field has either zero, one, or infinitely many points.

Finally, we provide some applications of our results to gradient and Hamiltonian $C^{1}$ vector fields. In particular, we also give a sufficient condition (Theorem $\mathrm{C}$ ) for a vector field to be a global Hamiltonian center. This result turns out to be a generalisation of 12 to the case in which $X$ is not necessarily a local diffeomorphism.

\section{Statement of the Results}

Throughout this paper, "for almost all" or "a.e." means "for Lebesgue almost all". We say that $p \in \mathbb{R}^{2}$ is a simple singularity of a $C^{1}$ vector field $X: \mathbb{R}^{2} \rightarrow \mathbb{R}^{2}$ if $X(p)=0$ and $\operatorname{Det}(D X(p)) \neq 0$. We say that $p \in \mathbb{R}^{2}$ is a hyperbolic singularity of $X$ if $X(p)=0$ and the eigenvalues of $D X(p)$ have non-null real parts. By "topologically equivalent" we understand "globally topologically equivalent". For the sake of simplicity we assume, whenever the singularity set of the vector field $X$ is not empty, that $X(0)=0$.

The main results of this paper are the following:

Theorem A. Let $X: \mathbb{R}^{2} \rightarrow \mathbb{R}^{2}$ be a $C^{1}$ vector field whose Jacobian matrix $D X(p)$ is Hurwitz for almost all $p \in \mathbb{R}^{2}$. If $0 \in \mathbb{R}^{2}$ is a hyperbolic singularity of $X$, then $X$ is topologically equivalent to the radial vector field $(x, y) \mapsto(-x,-y)$.

Theorem B. Let $X: \mathbb{R}^{2} \rightarrow \mathbb{R}^{2}$ be a $C^{1}$ vector field whose Jacobian matrix $D X(p)$ is Hurwitz for almost all $p \in \mathbb{R}^{2}$. Then the singularity set of $X$ is either an empty set, a one-point set, or a non-discrete set.

We say that a complex number $z \in \mathbb{C}$ is purely imaginary if $z=b i$ for some real number $b \neq 0$. Given $\epsilon \in\{-1,1\}$, we call the vector field $(x, y) \mapsto \epsilon(-y, x)$ a linear center.

Theorem C. Let $X: \mathbb{R}^{2} \rightarrow \mathbb{R}^{2}$ be a $C^{1}$ vector field whose Jacobian matrix $D X(p)$ has purely imaginary eigenvalues for almost all $p \in \mathbb{R}^{2}$. If $0 \in \mathbb{R}^{2}$ is a simple singularity of $X$, then $X$ is topologically equivalent to a linear center.

We also include the following interesting examples (see the proof in Section 7 ):

\section{Proposition 2.1.}

(a) There exists a non-injective and globally asymptotically stable $C^{1}$ vector field $X: \mathbb{R}^{2} \rightarrow \mathbb{R}^{2}$ whose Jacobian matrix $D X(p)$ is Hurwitz for almost all $p \in \mathbb{R}^{2}$.

(b) There exists a $C^{1}$ vector field $Y: \mathbb{R}^{2} \rightarrow \mathbb{R}^{2}$ whose Jacobian matrix $D Y(p)$ is Hurwitz for almost all $p \in \mathbb{R}^{2}$ and whose singularity set is a line ( $a$ non-discrete set).

To explain these results, let us fix the following notation. We say that a $C^{1}$ vector field $X: \mathbb{R}^{2} \rightarrow \mathbb{R}^{2}$ is Hurwitz (respectively Hurwitz a.e.) if its Jacobian matrix $D X(p)$ is Hurwitz for all $p \in \mathbb{R}^{2}$ (respectively for almost all $p \in \mathbb{R}^{2}$ ).

If the hypothesis of Hurwitz of Theorem 1.1 is replaced by Hurwitz a.e., one can no longer ensure that 0 is a global attractor of $X$. In fact, we present an 
example of a vector field Hurwitz a.e. which has the line $\left\{(x, y) \in \mathbb{R}^{2} \mid x=-1\right\}$ as its singularity set. However, if we rule out exotic behaviour by requiring that 0 is a hyperbolic singularity of $X$, then the hypothesis of Hurwitz a.e. still implies that 0 is a global attractor (Theorem A).

Theorem B characterizes the singularity set of a Hurwitz a.e. vector field: it has either zero, or one, or infinitely many points. In particular, a vector field having exactly two singularities cannot be Hurwitz a.e.

Theorem $\mathrm{C}$ gives spectral conditions for a $C^{1}$ vector field to be a global Hamiltonian center. It was proved in [12] that if a differentiable vector field $X: \mathbb{R}^{2} \rightarrow \mathbb{R}^{2}$ has the property that $X(0)=0$ and $D X(p)$ has purely imaginary eigenvalues for all $p \in \mathbb{R}^{2}$, then $X$ is topologically equivalent to a linear center. Theorem $\mathrm{C}$ says that this result is still true for a vector field whose Jacobian matrix $D X(p)$ has purely imaginary eigenvalues for almost all $p \in \mathbb{R}^{2}$, provided $0 \in \mathbb{R}^{2}$ is a simple singularity of $X$.

Some applications (Corollaries 6.1 and 6.2) of the main theorems to gradient and Hamiltonian $C^{1}$ vector fields are also included. A consequence of these corollaries is that the Jacobian determinant of a gradient (or Hamiltonian) $C^{1}$ vector field that has more than one singularity changes sign or vanishes on a set of positive Lebesgue measure. This is exploited in the section of examples.

In addition to the articles mentioned so far, we would like to attach some important historical remarks on the theme of global asymptotic stability and injectivity. Meisters-Olech [8] gave an affirmative answer to the Markus-Yamabe Problem (in the plane) in the polynomial case. In [9], Olech pointed out the relations between global asymptotic stability and global injectivity. The problem remained open for more than 30 years until it was completely solved by Gutierrez [4, Feßler [1] and Glutsyuk 3 in an affirmative way for $C^{1}$ vector fields. A generalisation of this result for differentiable (not necessarily $C^{1}$ ) vector fields was given by Fernandes, Gutierrez, and Rabanal [2] (see also [5]). Concerning the dynamics of planar Hamiltonian vector fields, we refer the reader to the article of Jarque and Nitecki [6].

\section{Vector fields with Hurwitz Jacobian matrix a.e.}

Given a $C^{1}$ vector field $X: \mathbb{R}^{2} \rightarrow \mathbb{R}^{2}$, let

$$
\operatorname{Spc}(X)=\left\{\text { Eigenvalues of } D X(p) \mid p \in \mathbb{R}^{2}\right\} \subset \mathbb{C} .
$$

The following theorem is the main injectivity result of [2] (see also [13]).

Theorem 3.1. Let $X: \mathbb{R}^{2} \rightarrow \mathbb{R}^{2}$ be a differentiable map. If $\operatorname{Spc}(X) \cap[0, \epsilon)=\emptyset$ for some $\epsilon>0$, then $X$ is injective.

Proposition 3.2. Let $X: \mathbb{R}^{2} \rightarrow \mathbb{R}^{2}$ be a $C^{1}$ vector field such that $D X(p)$ is Hurwitz for almost all $p \in \mathbb{R}^{2}$. Then the set $U=\left\{p \in \mathbb{R}^{2} \mid \operatorname{Det}(D X(p))>0\right\}$ is open and dense, and $\left.X\right|_{U}$ is injective.

Proof. For almost all $p \in \mathbb{R}^{2}, D X(p)$ is Hurwitz; thus Trace $(D X(p))<0$ and $\operatorname{Det}(D X(p))>0$. Now by the continuity of the partial derivatives of $X$, we have that $U$ is open and dense. Moreover, Trace $(D X(p)) \leq 0$ and $\operatorname{Det}(D X(p)) \geq 0$ for all $p \in \mathbb{R}^{2}$. Set $\epsilon_{n}=\frac{1}{n}$ and $X_{n}=X-\epsilon_{n} I$ ( $I$ is the identity map). Then

$$
\operatorname{Spc}\left(X_{n}\right) \subset\{z \in \mathbb{C} \mid \operatorname{Re}(z)<0\}
$$

for every integer $n \geq 1$, where $\operatorname{Re}(z)$ stands for the real part of the complex number $z$. By Theorem 3.1, we have that $X_{n}$ is injective for every integer $n \geq 1$. 
We claim that $\left.X\right|_{U}$ is injective. Otherwise, there exist $p, q \in U, p \neq q$, such that $X(p)=y=X(q)$. In this case, by the definition of $U$ and by the Inverse Function Theorem, there exist neighborhoods $U_{1}, U_{2} \subset U$ and $V \subset \mathbb{R}^{2}$ of $p, q$ and $y$, respectively, such that $\left.X\right|_{U_{i}}: U_{i} \rightarrow V$ is a homeomorphism and $U_{1} \cap U_{2}=\emptyset$. By the definition of $X_{n}$, for $n$ large enough, $X_{n}\left(U_{1}\right) \cap X_{n}\left(U_{2}\right)$ will contain a neighborhood $W$ of $y$. Hence, for all $w \in W$ and $n$ large enough, $\#\left(X_{n}^{-1}(w)\right) \geq 2$, where \# denotes the cardinality of the set. This contradicts the injectivity of $X_{n}$. Hence, the claim is true.

The singularity set of a $C^{1}$ vector field $X: \mathbb{R}^{2} \rightarrow \mathbb{R}^{2}$ is the set

$$
\operatorname{Sing}(X)=\left\{p \in \mathbb{R}^{2} \mid X(p)=0\right\} .
$$

Corollary 3.3. Let $X: \mathbb{R}^{2} \rightarrow \mathbb{R}^{2}$ be a $C^{1}$ vector field such that $D X(p)$ is Hurwitz for almost all $p \in \mathbb{R}^{2}$. If $0 \in \mathbb{R}^{2}$ is a hyperbolic singularity of $X$, then for all $\rho>0$ there exists $\epsilon>0$ such that $\|X(p)\|>\epsilon$ for all $\|p\|>\rho$. In particular, $\operatorname{Sing}(X)=\{0\}$.

Proof. By the hypotheses on $X$ and by the continuity of $p \mapsto \operatorname{Det}(D X(p))$, we have that $\operatorname{Det}(D X(0))>0$. Let $U=\left\{p \in \mathbb{R}^{2} \mid \operatorname{Det}(D X(p))>0\right\}$; thus $0 \in U$. It suffices to prove the claim for arbitrarily small $\rho>0$. Let $\rho>0$ be such that $B_{\rho} \subset U$, where $B_{\rho}$ is an open ball of radius $\rho$ centered at 0 . Because $\left.X\right|_{U}$ is an open map and $X(0)=0$, there exists a ball $B_{2 \epsilon}$ of radius $2 \epsilon$ centered at 0 such that $B_{2 \epsilon} \subset X\left(B_{\rho}\right)$. By Proposition 3.2, $U$ is an open, dense set, and $\left.X\right|_{U}$ is injective; thus $X\left(U \backslash B_{\rho}\right) \cap B_{2 \epsilon}=\emptyset$. Hence, $\|X(p)\| \geq 2 \epsilon$ for all $p \in U \backslash B_{\rho}$. We claim that $\|X(p)\|>\epsilon$ for all $p$ such that $\|p\|>\rho$. Let $p \in \mathbb{R}^{2}$ be such that $\|p\|>\rho$; then $p \in \mathbb{R}^{2} \backslash \overline{B_{\rho}}$. By the density of $U$, there exists a sequence $\left\{p_{n}\right\}_{n=1}^{\infty} \subset U \backslash \overline{B_{\rho}}$ tending to $p$ such that $\|X(p)\|=\lim _{n \rightarrow \infty}\left\|X\left(p_{n}\right)\right\|$. As $p_{n} \in U \backslash \overline{B_{\rho}}$, we have $\left\|X\left(p_{n}\right)\right\| \geq 2 \epsilon$ for all $n \geq 1$, hence $\|X(p)\| \geq 2 \epsilon>\epsilon$.

Given a $C^{1}$ vector field $X=(f, g): \mathbb{R}^{2} \rightarrow \mathbb{R}^{2}$, let $X^{*}=(-g, f)$ be the orthogonal vector field to $X$. The same notation as that for intervals of $\mathbb{R}$ will be used for oriented arcs of trajectory $[p, q],[p, q), \ldots$ (respectively $\left.[p, q]^{*},[p, q)^{*}, \ldots\right)$ of $X$ (respectively $X^{*}$ ), connecting the points $p$ and $q$. The orientation of theses arcs is that induced by $X$ (respectively $\left.X^{*}\right)$.

A compact rectangle $R=R\left(p_{1}, p_{2} ; q_{1}, q_{2}\right) \subset \mathbb{R}^{2}$ of a $C^{1}$ vector field $X: \mathbb{R}^{2} \rightarrow \mathbb{R}^{2}$ is the compact region whose boundary is a Jordan curve made up of two arcs of trajectory $\left[p_{1}, p_{2}\right],\left[q_{1}, q_{2}\right]$ of $X$ and two arcs of trajectory $\left[p_{1}, q_{1}\right]^{*},\left[p_{2}, q_{2}\right]^{*}$ of $X^{*}$. We assume that the flow induced by $X$ goes into $R$ by $\left[p_{1}, q_{1}\right]^{*}$ and leaves out $R$ by $\left[p_{2}, q_{2}\right]^{*}$. The points $p_{1}, p_{2}, q_{1}, q_{2}$ are the vertices of the rectangle $R$.

For any arc of trajectory $[p, q]^{*}$ of $X^{*}$, let

$$
L\left([p, q]^{*}\right)=\left|\int_{[p, q]^{*}}\|X\| d s\right|,
$$

where $d s$ denotes the arc length element. Given an arc of trajectory $[p, q]$ (respectively $\left.[p, q]^{*}\right)$, we denote by $\ell([p, q])$ (respectively $\ell\left([p, q]^{*}\right)$ ) its arc length.

Lemma 3.4. Let $R=R\left(p_{1}, p_{2} ; q_{1}, q_{2}\right) \subset \mathbb{R}^{2}$ be a compact rectangle of a $C^{1}$ vector field $X: \mathbb{R}^{2} \rightarrow \mathbb{R}^{2}$. Then

$$
L\left(\left[p_{2}, q_{2}\right]^{*}\right)-L\left(\left[p_{1}, q_{1}\right]^{*}\right)=\int_{R} \operatorname{Trace}(D X) d x \wedge d y .
$$


Proof. See Green's Formula as presented in [11, Corollary 5.7].

Let $\gamma_{p}^{+}$denote a solution of $u^{\prime}=X(u), u(0)=p$, with right-maximal existence interval $\left[0, b_{+}\right)$, where $b_{+} \in[0,+\infty) \cup\{+\infty\}$. We identify $\gamma_{p}^{+}$with its image $\gamma_{p}^{+}\left(\left[0, b_{+}\right)\right)$, and we call $\gamma_{p}^{+}$a positive semitrajectory of $X$. Given $q \in \mathbb{R}^{2}$, we set $d\left(q, \gamma_{p}^{+}\right)=\inf \left\{\left\|q-\gamma_{p}^{+}(t)\right\|: t \in\left[0, b_{+}\right)\right\}$. We denote by $\omega(p)$ the $\omega$-limit set of a point $p$,

$$
\omega(p)=\left\{q \in \mathbb{R}^{2} \mid \exists t_{n} \rightarrow b_{+} \text {such that } \lim _{n \rightarrow \infty} \gamma_{p}^{+}\left(t_{n}\right)=q\right\} .
$$

An alternative proof of the next proposition may be found in [2, Lemma 3.4, p. 480]. A complete proof is provided here.

Proposition 3.5. Let $X: \mathbb{R}^{2} \rightarrow \mathbb{R}^{2}$ be a $C^{1}$ vector field whose Jacobian matrix $D X(p)$ is Hurwitz for almost all $p \in \mathbb{R}^{2}$. If $0 \in \mathbb{R}^{2}$ is a hyperbolic singularity of $X$, then the set $\left\{p \in \mathbb{R}^{2} \mid \omega(p)=\emptyset\right\}$ is open.

Proof. Let $p_{1} \in \mathbb{R}^{2}$ be such that $\omega\left(p_{1}\right)=\emptyset$. Thus for some $0<K<\frac{\left\|p_{1}\right\|}{4}$ we have that $\gamma_{p_{1}}^{+} \subset \mathbb{R}^{2} \backslash B_{2 K}$, where $B_{2 K}=\left\{p \in \mathbb{R}^{2}:\|p\|<2 K\right\}$. We will prove that $\omega(p)=\emptyset$ for all $p$ in a neighborhood of $p_{1}$. Let

$$
V=\left\{q \in \mathbb{R}^{2} \mid d\left(q, \gamma_{p_{1}}^{+}\right)<K / 4\right\} .
$$

Notice that $V$ is a neighborhood of $\gamma_{p_{1}}^{+}$such that $\bar{V} \subset \mathbb{R}^{2} \backslash B_{K}$. Let $W$ be the union of all arcs of trajectory of $X^{*}$ intersecting $\gamma_{p_{1}}^{+} \backslash\left\{p_{1}\right\}$ and having arc length less than $K / 4$. By the Long Tubular Flow Theorem [10, Proposition 1.1, p. 93], $W$ is an open neighborhood of $\gamma_{p_{1}}^{+} \backslash\left\{p_{1}\right\}$. Besides, $W \subset V$. By Corollary 3.3, there exists $\epsilon>0$ such that $\|X(p)\|>\epsilon$ for all $p \in \mathbb{R}^{2} \backslash B_{K}$. Let $\left(x_{1}, y_{1}\right)^{*} \subset \bar{W} \subset \bar{V} \subset \mathbb{R}^{2} \backslash B_{K}$ be an arc of trajectory of $X^{*}$ containing $p_{1}$ so small that $\frac{d_{1}}{\epsilon} \ell\left(\left[x_{1}, y_{1}\right]^{*}\right)<\frac{K}{8}$, where $d_{1}=\sup \left\{\|X(z)\|: z \in\left[x_{1}, y_{1}\right]^{*}\right\}$. We claim that $\gamma_{q_{1}}^{+} \subset V$ for all $q_{1} \in\left[x_{1}, y_{1}\right]^{*}$. Suppose that the claim is false. So, without loss of generality, we may assume that $\gamma_{q_{1}}^{+} \not \subset V$ for some $q_{1} \in\left[p_{1}, y_{1}\right]^{*}$. Then there exists $\eta>0$ such that $\gamma_{q_{1}}^{+}(t) \in W$ for all $t \in(0, \eta)$ but $q_{2}:=\gamma_{q_{1}}^{+}(\eta) \notin W$. In this way, $\left(q_{1}, q_{2}\right) \subset W$. By the definition of $W$, for all $t \in(0, \eta)$ there exist $p(t) \in \gamma_{p_{1}}^{+}$and $q(t):=\gamma_{q_{1}}^{+}(t)$ such that $p_{1}, p(t), q_{1}, q(t)$ are vertices of the rectangle $R=R\left(p_{1}, p(t) ; q_{1}, q(t)\right)$. By Lemma 3.4. for the rectangle $R$ we have

$$
L\left([p(t), q(t)]^{*}\right)-L\left(\left[p_{1}, q_{1}\right]^{*}\right)=\int_{R} \operatorname{Trace}(D X) d x \wedge d y<0 .
$$

As $R \subset \bar{V} \subset \mathbb{R}^{2} \backslash B_{K}$ and $\|X(p)\|>\epsilon$ for all $p \in \mathbb{R}^{2} \backslash B_{K}$, we have that

$$
\begin{aligned}
\epsilon \ell\left([p(t), q(t)]^{*}\right) & \leq\left|\int_{[p(t), q(t)]^{*}}\|X\| d s\right|=L\left([p(t), q(t)]^{*}\right)<L\left(\left[p_{1}, q_{1}\right]^{*}\right) \\
& =\left|\int_{\left[p_{1}, q_{1}\right]^{*}}\|X\| d s\right| \leq d_{1} \ell\left(\left[p_{1}, q_{1}\right]^{*}\right) .
\end{aligned}
$$

Therefore, for all $t \in(0, \eta)$, we have that

$$
\ell\left([p(t), q(t)]^{*}\right)<\frac{d_{1}}{\epsilon} \ell\left(\left[p_{1}, q_{1}\right]^{*}\right) \leq \frac{d_{1}}{\epsilon} \ell\left(\left[x_{1}, y_{1}\right]^{*}\right)<\frac{K}{8} .
$$

We have shown above that for each $t \in(0, \eta),[p(t), q(t)]^{*}$ is an arc of trajectory of $X^{*}$ of arc length less than $K / 8$. It is easy to see that there exists $p_{2} \in \gamma_{p_{1}}^{+}$such that $\lim _{t \rightarrow \eta^{-}} p(t)=p_{2}$. It follows from (3.1) that there exists an arc of trajectory of $X^{*}$ 
with endpoints at $p_{2}$ and $q_{2}$, denoted by $\left[p_{2}, q_{2}\right]^{*}$, such that $\ell\left(\left[p_{2}, q_{2}\right]^{*}\right) \leq \frac{K}{8}<\frac{K}{4}$. Thus $\left[p_{2}, q_{2}\right]^{*} \subset W$, which contradicts the fact that $q_{2} \notin W$; thus the claim it true. In this way, $\gamma_{q_{1}}^{+} \subset V$ for all $q_{1} \in\left[x_{1}, y_{1}\right]^{*}$. By Corollary 3.3. Sing $(X)=\{0\}$. Since $\bar{V} \subset \mathbb{R}^{2} \backslash B_{K}$, we have that $V$ contains no singularity of $X$. By index theory, $\bar{V}$ contains no periodic trajectory of $X$. The Poincaré-Bendixson Theorem together with the fact that $\gamma_{q_{1}}^{+} \subset V$ imply that $\omega\left(q_{1}\right)=\emptyset$ for all $q_{1} \in\left[x_{1}, y_{1}\right]^{*}$. Hence, by the Long Tubular Flow Theorem, if we take a small ball $B\left(p_{1}\right) \subset V$ centered at $p_{1}$, all the trajectories $\gamma_{p}^{+}$, with $p \in B\left(p_{1}\right)$, will cross the transverse segment $\left[x_{1}, y_{1}\right]^{*}$. Thus $\omega(p)=\emptyset$ for all $p \in B\left(p_{1}\right)$.

Theorem A. Let $X: \mathbb{R}^{2} \rightarrow \mathbb{R}^{2}$ be a $C^{1}$ vector field whose Jacobian matrix $D X(p)$ is Hurwitz for almost all $p \in \mathbb{R}^{2}$. If $0 \in \mathbb{R}^{2}$ is a hyperbolic singularity of $X$, then $X$ is topologically equivalent to the radial vector field $(x, y) \mapsto(-x,-y)$.

Proof. By Corollary 3.3, $0 \in \mathbb{R}^{2}$ is the unique singularity of $X$. Let

$$
W^{s}=\left\{p \in \mathbb{R}^{2} \mid \omega(p)=\{0\}\right\} .
$$

It follows from the hypotheses that the eigenvalues of $D X(0)$ have negative real parts. Thus by the Hartman-Grobman Theorem, $X$ is locally topologically equivalent to the linear vector field $(x, y) \mapsto(-x,-y)$. This together with the Long Flow Box Theorem implies that $W^{s}$ is open. The hypothesis that $D X(p)$ is Hurwitz yields Trace $(D X(p))<0$ for almost all $p \in \mathbb{R}^{2}$, thus by Green's Formula $X$ has no periodic trajectories. In this way, by the Poincaré-Bendixson Theorem, for each $p \in \mathbb{R}^{2}$ either $\omega(p)=\{0\}$ or $\omega(p)=\emptyset$. Hence, $\mathbb{R}^{2} \backslash W^{s}=\left\{p \in \mathbb{R}^{2} \mid \omega(p)=\emptyset\right\}$. By Proposition 3.5, $\mathbb{R}^{2} \backslash W^{s}$ is open. Therefore, $W^{s}$ is an open and closed subset of $\mathbb{R}^{2}$, and so $W^{s}=\mathbb{R}^{2}$. To prove that $X$ is topologically equivalent to $(x, y) \mapsto(-x,-y)$ we have to construct a transverse circle to $X$, proceeding as in [5].

\section{Singularity Set of Hurwitz a.e. Vector Fields}

In this section we will prove the following theorem.

Theorem B. Let $X: \mathbb{R}^{2} \rightarrow \mathbb{R}^{2}$ be a $C^{1}$ vector field whose Jacobian matrix $D X(p)$ is Hurwitz for almost all $p \in \mathbb{R}^{2}$. Then the singularity set of $X$ is either an empty set, a one-point set, or a non-discrete set.

Proof. Let $S=\operatorname{Sing}(X)$. Suppose that $S$ is discrete. We have to show that $S$ is either empty or a one-point set. Assume that $S$ is non-empty. Consequently, we may write $S=\left\{z_{i}\right\}$, where $X\left(z_{i}\right)=0$ for all $i$. The claims below show that under the above hypotheses, $S$ is a one-point set.

Claim 1. Let $z \in S$ be fixed. There exist a sequence $\left\{X_{k}\right\}_{k=1}^{\infty}$ of $C^{1}$ vector fields tending to $X$ in the $C^{1}$ uniform topology and a sequence of points $\left\{v_{k}\right\}_{k=1}^{\infty} \subset \mathbb{R}^{2} \backslash S$ tending to $z$ such that $v_{k}$ is a hyperbolic singularity of $X_{k}$ for every integer $k \geq$ 1. Furthermore, each $X_{k}$ is Hurwitz a.e., that is, its Jacobian matrix $D X_{k}(p)$ is Hurwitz for almost all $p \in \mathbb{R}^{2}$.

Proof of Claim 1. For $u \in \mathbb{R}^{2}$ and $r>0$, set $B(u, r)=\left\{p \in \mathbb{R}^{2}:\|p-u\|<r\right\}$. For each integer $k \geq 1$, by the continuity of $X$ and because $X(z)=0$, there exists $n_{k}>k$ such that $X\left(B\left(z, \frac{1}{n_{k}}\right)\right) \subset B\left(0, \frac{1}{k}\right)$. Let $v_{k} \in B\left(z, \frac{1}{n_{k}}\right) \backslash S$ be such that $D X\left(v_{k}\right)$ is Hurwitz. Set $\omega_{k}=X\left(v_{k}\right)$. Notice that $\left\|\omega_{k}\right\|=\left\|X\left(v_{k}\right)\right\|<\frac{1}{k}$ and $\left\|v_{k}-z\right\|<\frac{1}{n_{k}}<\frac{1}{k}$. Set $X_{k}=X-\omega_{k}$. It is clear that $X_{k}\left(v_{k}\right)=0$ and that 
$D X_{k}\left(v_{k}\right)=D X\left(v_{k}\right)$ is Hurwitz; thus $v_{k}$ is a hyperbolic singularity of $X_{k}$. As $\left\|X(p)-X_{k}(p)\right\|=\left\|w_{k}\right\|<\frac{1}{k}$ for all $p \in \mathbb{R}^{2}$, we have that $X_{k}$ tends to $X$ in the $C^{1}$ uniform topology. Moreover, $D X_{k}(p)=D X(p)$ for all $p \in \mathbb{R}^{2}$. Hence, $D X_{k}(p)$ is Hurwitz for almost all $p \in \mathbb{R}^{2}$. Notice that $v_{k} \rightarrow z$ as $k \rightarrow \infty$.

Claim 2. Each singularity $z \in S$ has index 1 .

Proof of Claim 2. Let $z \in S$ be fixed. Let $\left\{X_{k}\right\}_{k=1}^{\infty}$ and $\left\{v_{k}\right\}_{k=1}^{\infty}$ be as in Claim 1 . Given a topological circle $\gamma$ contained in $\mathbb{R}^{2}$, we call the open bounded connected component of $\mathbb{R}^{2} \backslash \gamma$ the interior of $\gamma$ and denote it by $\operatorname{Int}(\gamma)$. Now let $\gamma \subset \mathbb{R}^{2}$ be a topological circle such that $z \in \operatorname{Int}(\gamma)$ and $\overline{\operatorname{Int}(\gamma)} \cap(S \backslash\{z\})=\emptyset$. As $v_{k} \rightarrow z$ as $k \rightarrow \infty$, we may assume that $v_{k} \in \operatorname{Int}(\gamma)$ for all $k$. By Theorem $\mathrm{A}$, we have that each $X_{k}$ is globally asymptotically stable. In this way, the index of $X_{k}$ along $\gamma$ is equal to 1 . By continuity, as $X_{k} \rightarrow X$ uniformly, we have that the index of $X$ along $\gamma$ is 1 . As $z$ is the only singularity of $X$ contained in Int $(\gamma)$, we have that its index is 1 .

Claim 3. $S$ is a one-point set.

Proof of Claim 3. Suppose that $S$ has at least two singularities, say $z_{1}$ and $z_{2}$. Let $\gamma$ be a topological circle such that $\left\{z_{1}, z_{2}\right\} \subset \operatorname{Int}(\gamma)$ and $\overline{\operatorname{Int}(\gamma)} \cap\left(S \backslash\left\{z_{1}, z_{2}\right\}\right)=\emptyset$. We can use the reasoning in the proof of Claim 2 to show that the index of the vector field $X$ along $\gamma$ is 1 . This contradicts Claim 2, which states that $\operatorname{Int}(\gamma)$ contains exactly two singularities of index 1 . So $S$ is a one-point set and the proof is finished.

\section{VECTOR FIELDS WITH NON-HYPERBOLIC JACOBIAN MATRIX A.E.}

In this section we will prove Theorem C. We will need some lemmas.

Proposition 5.1. Let $X: \mathbb{R}^{2} \rightarrow \mathbb{R}^{2}$ be a $C^{1}$ vector field whose Jacobian matrix $D X(p)$ has purely imaginary eigenvalues for almost all $p \in \mathbb{R}^{2}$. Then the set $U=\left\{p \in \mathbb{R}^{2} \mid \operatorname{Det}(D X(p))>0\right\}$ is open and dense, and $\left.X\right|_{U}$ is injective.

Proof. The proof of Proposition 3.2 holds verbatim. The only change is to replace Trace $(D X(p))<0$ by Trace $(D X(p))=0$ a.e.

Corollary 5.2. Let $X: \mathbb{R}^{2} \rightarrow \mathbb{R}^{2}$ be a $C^{1}$ vector field whose Jacobian matrix $D X(p)$ has purely imaginary eigenvalues for almost all $p \in \mathbb{R}^{2}$. If $0 \in \mathbb{R}^{2}$ is a simple singularity of $X$, then for all $\rho>0$ there exists $\epsilon>0$ such that $\|X(p)\|>\epsilon$ for all $\|p\|>\rho$. In particular, $\operatorname{Sing}(X)=\{0\}$.

Proof. It follows from Proposition 5.1. The proof is as in Corollary 3.3

We say that a $C^{1}$ vector field $X: \mathbb{R}^{2} \rightarrow \mathbb{R}^{2}$, with $X(0)=0$, is locally topologically equivalent to a linear center $L: \mathbb{R}^{2} \rightarrow \mathbb{R}^{2}$ around 0 if there exist neighborhoods $U, V$ of $0 \in \mathbb{R}^{2}$ and a homeomorphism $h: U \rightarrow V$ that takes trajectories of $\left.X\right|_{U}$ onto trajectories of $\left.L\right|_{V}$.

Lemma 5.3. Let $X: \mathbb{R}^{2} \rightarrow \mathbb{R}^{2}$ be a $C^{1}$ vector field whose Jacobian matrix $D X(p)$ has purely imaginary eigenvalues for almost all $p \in \mathbb{R}^{2}$. If $0 \in \mathbb{R}^{2}$ is a simple singularity of $X$, then $X$ is locally topologically equivalent to a linear center around 0 . 
Proof. Let $X=(f, g)$, where $f, g: \mathbb{R}^{2} \rightarrow \mathbb{R}$ are the $C^{1}$ component functions of $X$. We claim that $X^{*}=(-g, f)$ is conservative, that is, there exists a $C^{2}$ function $h: \mathbb{R}^{2} \rightarrow \mathbb{R}$ such that $X^{*}=\nabla h=\left(h_{x}, h_{y}\right)$. In fact, the hypothesis that the Jacobian matrix is Hurwitz a.e. together with the continuity of $p \mapsto D X(p)$ yield Trace $(D X(p))=0$ for all $p \in \mathbb{R}^{2}$. Hence, $f_{x}(p)+g_{y}(p)=0$ for all $p \in \mathbb{R}^{2}$. In this way, $X^{*}$ is an irrotational $C^{1}$ vector field defined in a simply connected domain, thus $X^{*}$ is conservative. In other words, there exists a $C^{2}$ function $h: \mathbb{R}^{2} \rightarrow \mathbb{R}$ such that $X=\left(h_{y},-h_{x}\right)$, so $X$ is tangent to the level curves of $h$. Notice that the Hessian of $h$ satisfies

$$
H_{h}(p)=\left|\begin{array}{ll}
h_{x x}(p) & h_{x y}(p) \\
h_{y x}(p) & h_{y y}(p)
\end{array}\right|=\left|\begin{array}{cc}
h_{y x}(p) & h_{y y}(p) \\
-h_{x x}(p) & -h_{x y}(p)
\end{array}\right|=\operatorname{Det}(D X(p)) .
$$

By hypothesis, $\operatorname{Det}(D X(p))>0$ for almost all $p \in \mathbb{R}^{2}$. Moreover, since 0 is a simple singularity of $X$, Det $(D X(0)) \neq 0$. Hence, $H_{h}(0)=\operatorname{Det}(D X(0))>0$. Besides, 0 is a non-degenerate critical point of $h$. By the Morse Lemma, the level curves of $h$ in a neighborhood of 0 are, up to a change of coordinates, the level curves of the function $(x, y) \mapsto x^{2}+y^{2}$ in a neighborhood of 0 .

We say that a subset $C$ of $\mathbb{R}^{2}$ is a topological circle if there exists a homeomorphism $h: \mathbb{R}^{2} \rightarrow \mathbb{R}^{2}$ such that $h(C)$ is the unit circle $\mathbb{S}^{1}$. Given a topological circle $\gamma$, we let Int $(\gamma)$ denote the bounded connected component of $\mathbb{R}^{2} \backslash \gamma$. We say that $A \subset \mathbb{R}^{2}$ is an annulus if there exist topological circles $\gamma_{1}, \gamma_{2} \subset \mathbb{R}^{2}$ such that $\overline{\operatorname{Int}\left(\gamma_{1}\right)} \subset \operatorname{Int}\left(\gamma_{2}\right)$ and $A=\operatorname{Int}\left(\gamma_{2}\right) \backslash \overline{\operatorname{Int}\left(\gamma_{1}\right)}$. An annulus $A$ is periodic if $\gamma_{p}^{+}$is a periodic trajectory for all $p \in A$.

Lemma 5.4. Let $X: \mathbb{R}^{2} \rightarrow \mathbb{R}^{2}$ be a $C^{1}$ vector field whose Jacobian matrix $D X(p)$ has purely imaginary eigenvalues for almost all $p \in \mathbb{R}^{2}$. Suppose that $0 \in \mathbb{R}^{2}$ is a simple singularity of $X$. If $\gamma_{1}$ and $\gamma_{2}$ are periodic trajectories of $X$ satisfying $\overline{\operatorname{Int}\left(\gamma_{1}\right)} \subset \operatorname{Int}\left(\gamma_{2}\right)$, then $A=\operatorname{Int}\left(\gamma_{2}\right) \backslash \overline{\operatorname{Int}\left(\gamma_{1}\right)}$ is a periodic annulus.

Proof. Let $x \in A$. By Corollary [5.2. Sing $(X)=\{0\}$, and so $A$ contains no singularity of $X$. By the Poincaré-Bendixson Theorem, we have that $\omega(x)$ is a periodic trajectory. We claim that $\gamma_{x}^{+}$is periodic; otherwise there exist $p \in \omega(x)$ and an orthogonal section $\Sigma$ tangent to $X^{*}$ and passing through $p$ such that $\gamma_{x}^{+}$intersects $\Sigma$ monotonically and infinitely many times. Let $\left\{p_{n}\right\}_{n=0}^{\infty}$ be the corresponding sequence of intersection points, where $p_{n} \rightarrow p$ as $n \rightarrow \infty$. By Lemma 3.4 and because Trace $(D X(p))=0$ for almost all $p \in \mathbb{R}^{2}$, we have that for every integer $n \geq 1$,

$$
0<L\left(\left[p_{0}, p_{1}\right]^{*}\right)=L\left(\left[p_{n}, p_{n+1}\right]^{*}\right),
$$

which is a contradiction since $L\left(\left[p_{n}, p_{n+1}\right]^{*}\right) \rightarrow 0$ as $n \rightarrow \infty$. This proves the claim.

Theorem C. Let $X: \mathbb{R}^{2} \rightarrow \mathbb{R}^{2}$ be a $C^{1}$ vector field whose Jacobian matrix $D X(p)$ has purely imaginary eigenvalues for almost all $p \in \mathbb{R}^{2}$. If $0 \in \mathbb{R}^{2}$ is a simple singularity of $X$, then $X$ is topologically equivalent to a linear center.

Proof. The idea is to construct a sequence $A_{1}, A_{2}, A_{3}, \ldots$ of periodic annulus such that $\bigcup_{n=1}^{\infty} \overline{A_{n}}=\mathbb{R}^{2} \backslash\{0\}$. By Lemma 5.3, there exists a sequence $B_{1}, B_{2}, B_{3}, \ldots$ of periodic annulus such that $B=\bigcup_{n=1}^{\infty} \overline{B_{n}}$ is a punctured neighborhood of 0 . By 
Corollary [5.2. Lemma 5.4 and the proof of [12, Proposition 2.3, p. 656], there exists a sequence $C_{1}, C_{2}, C_{3}, \ldots$ of periodic annulus such that $\bigcup_{n=1}^{\infty} \overline{C_{n}}=\mathbb{R}^{2} \backslash(B \cup\{0\})$. In this way, $B_{1}, C_{1}, B_{2}, C_{2}, \ldots$ is a sequence of periodic annulus such that

$$
\bigcup_{n=1}^{\infty}\left(\overline{B_{n}} \cup \overline{C_{n}}\right)=\mathbb{R}^{2} \backslash\{0\} .
$$

\section{Corollaries of the main theorems}

We say that $X: \mathbb{R}^{2} \rightarrow \mathbb{R}^{2}$ is a Hamiltonian $C^{1}$ vector field if there exists a $C^{2}$ function $h: \mathbb{R}^{2} \rightarrow \mathbb{R}$ such that $X=\left(-h_{y}, h_{x}\right)$.

Corollary 6.1. Let $X: \mathbb{R}^{2} \rightarrow \mathbb{R}^{2}$ be a Hamiltonian $C^{1}$ vector field whose Jacobian determinant is positive almost everywhere. If $0 \in \mathbb{R}^{2}$ is a simple singularity of $X$, then $X$ is topologically equivalent to a linear center.

Proof. By hypothesis, there exists a $C^{2}$ function $h: \mathbb{R}^{2} \rightarrow \mathbb{R}$ such that $X=$ $\left(-h_{y}, h_{x}\right)$. By the Schwarz Lemma, the mixed partial derivatives of $h$ are equal to each other; that is, $h_{y x}=h_{x y}$. Hence, Trace $(D X(p))=-h_{y x}(p)+h_{x y}(p)=0$ for almost all $p \in \mathbb{R}^{2}$. By hypothesis, Det $(D X(p))>0$ for almost all $p \in \mathbb{R}^{2}$. Putting it all together, $D X(p)$ has purely imaginary eigenvalues for almost all $p \in \mathbb{R}^{2}$. Now the result follows from Theorem $\mathrm{C}$.

We say that $X: \mathbb{R}^{2} \rightarrow \mathbb{R}^{2}$ is a gradient $C^{1}$ vector field if there exists a $C^{2}$ function $h: \mathbb{R}^{2} \rightarrow \mathbb{R}$ such that $X=\left(h_{x}, h_{y}\right)$.

Corollary 6.2. Let $X: \mathbb{R}^{2} \rightarrow \mathbb{R}^{2}$ be a gradient $C^{1}$ vector field whose Jacobian determinant is positive almost everywhere. If $0 \in \mathbb{R}^{2}$ is a hyperbolic singularity of $X$, then $X$ is topologically equivalent to the radial vector field $(x, y) \mapsto(-x,-y)$.

Proof. Let $Y=R X$ be the vector field obtained from $X$ by the rotation $R: \mathbb{R}^{2} \rightarrow$ $\mathbb{R}^{2}$ defined by $R(x, y)=(-y, x)$. It is clear that $Y$ is a Hamiltonian vector field and that 0 is a simple singularity of $Y$. By Corollary 6.1, $Y$ is topologically equivalent to a linear center. This means that we can split $\mathbb{R}^{2} \backslash\{0\}$ as the union of transverse circles to $X$. It is not diffcult to show that $X$ is topologically equivalent to the radial vector field $(x, y) \mapsto(-x,-y)$.

\section{Commented examples}

In this section we present a study of the spectrum (i.e. the distribution of the eigenvalues of $D X(p)$ as $p$ runs in $\mathbb{R}^{2}$ ) of many maps and vector fields. We group the cases with the same behaviour into subsections.

7.1. Non-injective maps whose Jacobian matrix is Hurwitz almost everywhere. Table 1 presents three examples of non-injective planar maps. The first map will be used to prove the following proposition.

\section{Proposition 2.1.}

(a) There exists a non-injective and globally asymptotically stable $C^{1}$ vector field $X: \mathbb{R}^{2} \rightarrow \mathbb{R}^{2}$ whose Jacobian matrix $D X(p)$ is Hurwitz for almost all $p \in \mathbb{R}^{2}$.

(b) There exists a $C^{1}$ vector field $Y: \mathbb{R}^{2} \rightarrow \mathbb{R}^{2}$ whose Jacobian matrix $D Y(p)$ is Hurwitz for almost all $p \in \mathbb{R}^{2}$ and whose singularity set is a line ( $a$ non-discrete set). 
Proof. (a) Let $X: \mathbb{R}^{2} \rightarrow \mathbb{R}^{2}$ be the $C^{1}$ vector field defined by $X(x, y)=F(x, y)=$ $\left(-(x+1)^{3}+1,-(x+1)^{2}(y+1)+1\right)$. By Table 1 we have that $D X(x, y)$ is Hurwitz everywhere except for the line $\{(x, y) \mid x=-1\}$. Furthermore, $p=(0,0)$ is a hyperbolic singularity of $X$. It follows from Theorem A that $X$ is globally asymptotically stable. Moreover, $X$ (as a map) is not injective since it takes the line $\{(x, y) \mid x=-1\}$ onto the point $(1,1)$.

(b) The vector field $Y: \mathbb{R}^{2} \rightarrow \mathbb{R}^{2}$ defined by $Y(x, y)=\left(-x^{3},-x^{2} y\right)$ has the line $S=\{(x, y) \mid x=0\}$ as its singularity set. Moreover, $D Y(p)$ is Hurwitz for all $p \in \mathbb{R}^{2} \backslash S$. Of course, $Y$ is not globally asymptotically stable.

Olech [9] proved that, for planar $C^{1}$ vector fields whose Jacobian matrix is Hurwitz everywhere, injectivity of the vector field (considered as a map) and global asymptotically stability are equivalent. Proposition 2.1 shows that such correspondence is lost under the weaker hypothesis that the Jacobian matrix is Hurwitz almost everywhere.

TABLE 1. Non-injective maps with Hurwitz a.e. Jacobian matrix

\begin{tabular}{l|l|l} 
Map & Trace $(D F(x, y))$ & $\operatorname{Det}(D F(x, y))$ \\
\hline$F(x, y)=\left(-(x+1)^{3}+1,-(x+1)^{2}(y+1)+1\right)$ & $-4(x+1)^{2}$ & $3(x+1)^{4}$ \\
$G(x, y)=\left(\frac{-x^{3}}{1+x^{2}}, \frac{-y x^{2}}{1+x^{2}}\right)$ & $\frac{-2 x^{2}\left(2+x^{2}\right)}{\left(1+x^{2}\right)^{2}}$ & $\frac{x^{4}\left(3+x^{2}\right)}{\left(1+x^{2}\right)^{3}}$ \\
$H(x, y)=\left[-1+\frac{2}{\pi} \operatorname{Tan}^{-1}\left(\frac{y}{x}\right)\right] \cdot(x, y)$ & $-2+\frac{4}{\pi} \operatorname{Tan}^{-1}\left(\frac{y}{x}\right)$ & $\frac{\left(\pi-2 \operatorname{Tan}^{-1}\left(\frac{y}{x}\right)\right)^{2}}{\pi^{2}}$ \\
\hline
\end{tabular}

As for the other examples in Table $1, G$ is a rational map whose Jacobian matrix is Hurwitz everywhere except for the line $\{(x, y) \mid x=0\}$. This set is also the set of zeros of $G$. Notice that by Proposition 3.2 $G$ is injective on $\mathbb{R}^{2} \backslash\{(x, y) \mid x=0\}$.

The map $H$ can be continuously extended in a non-injective way to the whole plane. Its Jacobian matrix is Hurwitz almost everywhere. Considered as vector fields, $G$ and $H$ are not globally asymptotically stable because their singularity sets contain a line of singularities.

TABLE 2. Hamiltonian vector fields

\begin{tabular}{c|l} 
Vector field & \multicolumn{1}{c}{$\operatorname{Det}(D F(x, y))$} \\
\hline$X(x, y)=\left(-(y-1)^{3}-1,(x-1)^{3}+1\right)$ & $9(x-1)^{2}(y-1)^{2}$ \\
$Y(x, y)=\left(-2 e^{-\left(x^{2}+y^{2}\right)} x y,\left(2 x^{2}-1\right) e^{-\left(x^{2}+y^{2}\right)}\right)$ & $-4\left(2 x^{4}+y^{2}+x^{2}\left(-3+2 y^{2}\right)\right) e^{-2\left(x^{2}+y^{2}\right)}$ \\
$Z(x, y)=\left(-2 y+4 y^{3},-2 x+4 x^{3}\right)$ & $-4\left(-1+6 x^{2}\right)\left(-1+6 y^{2}\right)$ \\
\hline
\end{tabular}

7.2. Hamiltonian vector fields and their Jacobians. Corollary 6.1 may be applied to understand the phase portrait of Hamiltonian $C^{1}$ vector fields. In Table 2 three examples of Hamiltonian vector fields are displayed. The first one, $X$, has 


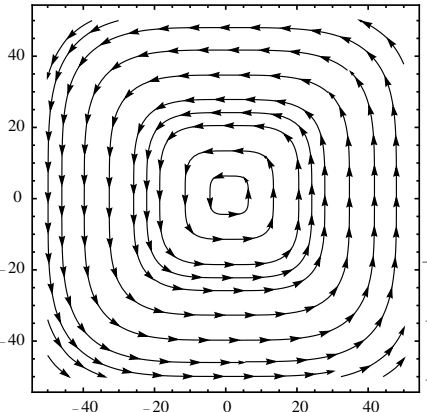

(A) Vector field $\mathrm{X}$

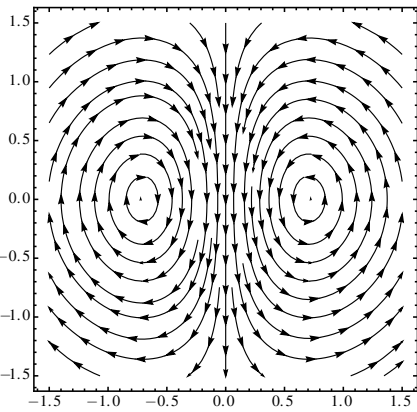

(B) Vector field Y

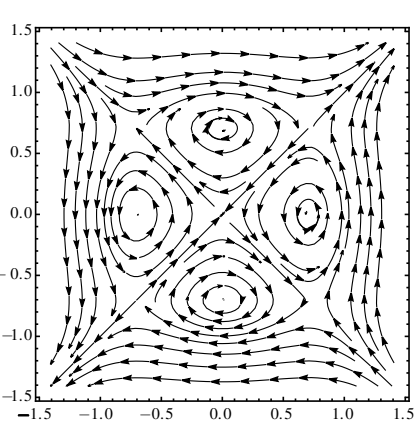

(c) Vector field Z

Figure 1. Phase portraits of Hamiltonian vector fields

the Jacobian positive almost everywhere. Besides, 0 is a simple singularity of $X$. In this way, by Corollary 6.1, $X$ is a global center (see Figure 17A). Concerning the vector fields $Y$ and $Z$, as each has more than one singularity, their Jacobian determinants have necessarily to change sign or to vanish on a set of positive Lebesgue measure (see Figures $1 \mathrm{~B}$ and $1 \mathrm{C}$ ). This prescription agrees with the formula of the corresponding Jacobian determinants (Table 2).

\section{ACKNOWLEDGMENTS}

This paper was developed during a visit of the authors to the Abdus Salam International Centre for Theoretical Physics (ICTP). The authors would like to thank the members of the Mathematics Section for their kind hospitality. The authors highly appreciate the comments and suggestions of the anonymous referee, which greatly improved the clarity of this paper.

\section{REFERENCES}

[1] Robert Feßler, A proof of the two-dimensional Markus-Yamabe stability conjecture and a generalization, Ann. Polon. Math. 62 (1995), no. 1, 45-74. MR1348217(96k:34099a)

[2] Alexandre Fernandes, Carlos Gutierrez, and Roland Rabanal, Global asymptotic stability for differentiable vector fields of $\mathbb{R}^{2}$, J. Differential Equations 206 (2004), no. 2, 470-482, DOI 10.1016/j.jde.2004.04.015. MR2096702 (2005h:37055)

[3] A. A. Glutsyuk, The asymptotic stability of the linearization of a vector field on the plane with a singular point implies global stability (Russian, with Russian summary), Funktsional. Anal. i Prilozhen. 29 (1995), no. 4, 17-30, 95, DOI 10.1007/BF01077471; English transl., Funct. Anal. Appl. 29 (1995), no. 4, 238-247 (1996). MR1375538 (96k:34099c)

[4] Carlos Gutiérrez, A solution to the bidimensional global asymptotic stability conjecture (English, with English and French summaries), Ann. Inst. H. Poincaré Anal. Non Linéaire 12 (1995), no. 6, 627-671. MR 1360540 (96k:34099b)

[5] Carlos Gutierrez, Benito Pires, and Roland Rabanal, Asymptotic stability at infinity for differentiable vector fields of the plane, J. Differential Equations 231 (2006), no. 1, 165-181, DOI 10.1016/j.jde.2006.07.025. MR2287882(2008a:34111)

[6] Xavier Jarque and Zbigniew Nitecki, Hamiltonian stability in the plane, Ergodic Theory Dynam. Systems 20 (2000), no. 3, 775-799, DOI 10.1017/S0143385700000419. MR.1764927 (2001d:37019)

[7] Lawrence Markus and Hidehiko Yamabe, Global stability criteria for differential systems, Osaka Math. J. 12 (1960), 305-317. MR.0126019 (23 \#A3316) 
[8] Gary Meisters and Czesław Olech, Solution of the global asymptotic stability Jacobian conjecture for the polynomial case, Analyse mathématique et applications, Gauthier-Villars, Montrouge, 1988, pp. 373-381. MR956968 (90b:58135)

[9] Czesław Olech, On the global stability of an autonomous system on the plane, Contributions to Differential Equations 1 (1963), 389-400. MR.0147734 (26 \#5248)

[10] Jacob Palis Jr. and Welington de Melo, Geometric theory of dynamical systems, An introduction, translated from the Portuguese by A. K. Manning. Springer-Verlag, New York, 1982. MR669541(84a:58004)

[11] Washek F. Pfeffer, The multidimensional fundamental theorem of calculus, J. Austral. Math. Soc. Ser. A 43 (1987), no. 2, 143-170. MR896622 (89b:26013)

[12] Roland Rabanal, Center type performance of differentiable vector fields in the plane, Proc. Amer. Math. Soc. 137 (2009), no. 2, 653-662, DOI 10.1090/S0002-9939-08-09686-X. MR2448587(2010a:34069)

[13] Roland Rabanal, On differentiable area-preserving maps of the plane, Bull. Braz. Math. Soc. (N.S.) 41 (2010), no. 1, 73-82. MR.2609212(2011e:26011)

Departamento de ComputaÇão e Matemática, Faculdade de Filosofia, Ciências e Letras, Universidade de SÃo PAUlo, 14040-901 Ribeirão Preto, SP, Brazil

E-mail address: benito@ffclrp.usp.br PERÚ

Sección Matemática, Pontificia Universidad Católica del Perú, San Miguel, Lima 32 ,

E-mail address: rrabanal@pucp.edu.pe 